International Journal of Pure and Applied Mathematics

Volume 107 No. 3 2016, 537-549

ISSN: 1311-8080 (printed version); ISSN: 1314-3395 (on-line version)

url: http://www.ijpam.eu

doi: 10.12732/ijpam.v107i3.3

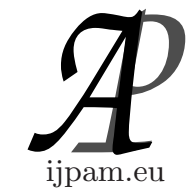

\title{
THEORY OF DISCRETE FOURIER SERIES GENERATED BY GENERALIZED DIFFERENCE OPERATOR
}

\author{
M. Maria Susai Manuel ${ }^{1}$, G. Dominic Babu ${ }^{2}$, G. Britto Antony Xavier ${ }^{3}$ \\ ${ }^{1}$ Department of Mathematics \\ R.M.D. Engineering College \\ Kavaraipettai, 601 206, Tamil Nadu, S. INDIA \\ ${ }^{2}$ Department of Mathematics \\ Annai Velankanni College \\ Tholaiyavattam, Kanyakumari District, Tamil Nadu, S. INDIA \\ ${ }^{3}$ Department of Mathematics \\ Sacred Heart College \\ Tirupattur, Vellore District, INDIA
}

\begin{abstract}
Constant amplitude transforms like Discrete Fourier Transform (DFT), Walsh transform, nonlinear phase Walsh-like transforms and Gold codes have been successfully used in many wire-line and wireless communication technologies including code division multiple access (CDMA), discrete multi-tone (DMT) and orthogonal frequency division multiplexing (OFDM) types. In this paper, we derive the discrete Fourier Series using orthonormal functions and generalized difference operator with its inverse. Suitable examples are provided to illustrate the main results.
\end{abstract}

AMS Subject Classification: 65B10, 65T10, 42A20

Key Words: discrete Fourier series, generalized difference operator, discrete orthonormal system

\section{Introduction}

In 1807, Fourier astounded some of his contemporaries by asserting that an "arbitrary" function could be expressed as a linear combination of sine and cosine

Received: January 5, 2016

Published: April 19, 2016

${ }^{\S}$ Correspondence author (c) 2016 Academic Publications, Ltd. url: www.acadpubl.eu 
functions. For a brief but excellent account of the history of this subject and its impact on the development of mathematics, one can refer $[1,2,3,4,5]$. These linear combinations, now called Fourier series, have become an indispensable tool in the analysis of certain periodic phenomena (such as vibrations, planetary and wave motion) which are applied in physics and engineering $[6,7,8,9]$.

In 1989, Miller and Rose [10] introduced the discrete analogue of the RiemannLiouville fractional derivative and proved some properties of the fractional difference operator. The general fractional h-difference Riemann- Liouville operator and its inverse $\Delta_{h}^{-\nu} f(t)$ were mentioned in $[11,12]$. As application of $\Delta_{h}^{-\nu}$, by taking $\nu=m$ (positive integer) and $h=\ell$, the sum of the $m^{\text {th }}$ partial sums on $n^{\text {th }}$ powers of arithmetic, arithmetic-geometric progressions and products of $\mathrm{n}$ consecutive terms of an arithmetic progression have been derived using $\Delta_{\ell}^{-m} u(k)$, where $\Delta_{\ell} u(k)=u(k+\ell)-u(k)[13]$.

The basic problems in the theory of discrete Fourier series are described in the setting of discrete orthogonal functions. Therefore, first we present some terminology concerning discrete orthogonal function and then we develop the theory of discrete Fourier series. Throughout this paper, we assume that the interval $I=[a, b], a<b, \ell=\frac{b-a}{N}, i=\sqrt{-1}$ and $N$ is a positive integer.

\section{Preliminaries}

An $n^{\text {th }}$ root of unity is a complex number satisfying the equation

$$
z^{N}=1, \quad N=0,1,2, \ldots
$$

If $z$ holds equation (1) but $z^{m} \neq 1 ; 0<m<N-1$, then $z$ is defined as a primitive $N^{t h}$ root of unity. The complex number $z_{0}=e^{j(2 \pi / N)}$, where $j^{2}=-1$, is the primitive $N^{\text {th }}$ root of unity with the smallest positive argument. The other primitive $N^{\text {th }}$ roots of unity are expressed as

$$
z_{n}=e^{j(2 \pi / N) n}, \quad n=1,2,3, \ldots, N-1,
$$

where $n$ and $N$ are co-prime. All $N^{t h}$ roots of unity satisfy the unique summation property of a geometric series expressed as

$$
\sum_{k=0}^{N-1} z_{n}^{k}=\left.\Delta^{-1} z_{n}^{k}\right|_{k=0} ^{N}=\frac{z_{n}^{N}-1}{z_{n}-1}= \begin{cases}1 & N=1 \\ 0 & N>1\end{cases}
$$

A periodic with period of $\mathrm{N}$, constant modulus, complex discrete-time sequence $e_{r}(k)$ is defined as

$$
e_{r}(k)=\left(z_{r}\right)^{k}=e^{j(2 \pi / N) r k}, \quad r, k=0,1,2, \ldots, N-1 .
$$


This complex sequence over a finite discrete time interval in a geometric series is expressed according to equation (3) as follows.

$$
\sum_{k=0}^{N-1} e_{r}(k)=\left.\Delta^{-1} e_{r}(k)\right|_{k=0} ^{N}=\left.\Delta^{-1} e^{j(2 \pi r / N) k}\right|_{k=0} ^{N}=\left\{\begin{array}{l}
N \quad \text { if } \quad r=m N \\
0 \quad \text { if } \quad r \neq m N
\end{array}\right.
$$

This mathematical property is utilized with the factorization into two orthogonal exponential functions, where one defines the Discrete Fourier Transform(DFT) $\left\{e_{n}(k)\right\}$ satisfying

$$
\left.\Delta^{-1} e_{n}(k) e_{m}^{*}(k)\right|_{k=0} ^{N}=\left.\Delta^{-1} e^{j(2 \pi / N)(n-m)(k)}\right|_{k=0} ^{N}=\left\{\begin{array}{l}
N \quad \text { if } n-m=r=m N \\
0 \quad \text { if } n-m=r \neq m N
\end{array}\right.
$$

where $m, n$ are integers and the notation $(*)$ represents the complex conjugate of a function. The equation (6) motivates us to define the generalized discrete orthonormal system and Fourier series by replacing $\Delta$ by $\Delta_{\ell}$ and $e_{n}(k)$ by $u_{n}(k)$.

Definition 2.1. [14] Let $u(k), k \in[0, \infty)$, be a real or complex valued function and $\ell \in(0, \infty)$. Then, the generalized difference operator $\Delta_{\ell}$ on $u(k)$ is defined as

$$
\Delta_{\ell} u(k)=u(k+\ell)-u(k),
$$

and the inverse of $\Delta_{\ell}$ denoted by $\Delta_{\ell}^{-1}$ is defined as

$$
\text { if } \Delta_{\ell} v(k)=u(k), \text { then } v(k)=\Delta_{\ell}^{-1} u(k)+c,
$$

where $c$ is a constant.

Example 2.2. [14] If we denote $k_{\ell}^{(n)}=k(k-\ell) \cdots(k-(n-1) \ell)$ and $k=k_{\ell}^{(0)}$, then we have

$$
\Delta_{\ell}^{-1} k_{\ell}^{(n)}=\frac{k_{\ell}^{(n+1)}}{(n+1) \ell}+c
$$

Theorem 2.3. [14] For $k \in[a, b]$ if $\ell=\frac{b-a}{N}$, then we have

$$
\left.\Delta_{\ell}^{-1} u(k)\right|_{a} ^{b}=\sum_{r=1}^{N} u(b-r \ell)=\sum_{r=0}^{N-1} u(a+r \ell)
$$


Lemma 2.4. Let $p$ be real and $\ell>0$. If $1 \neq \cos p \ell$, then

$$
\Delta_{\ell}^{-1} \sin p k=\frac{\sin p(k-\ell)-\sin p k}{2(1-\cos p \ell)}
$$

and

$$
\Delta_{\ell}^{-1} \cos p k=\frac{\cos p(k-\ell)-\cos p k}{2(1-\cos p \ell)} .
$$

Proof. Replacing $\mathrm{u}(\mathrm{k})$ by $\sin p k$ and $\cos p k$ in (7), we get

$$
\begin{aligned}
& \Delta_{\ell} \sin p k=(\cos p \ell-1) \sin p k+\sin p \ell \cos p k, \\
& \Delta_{\ell} \cos p k=(\cos p \ell-1) \cos p k-\sin p \ell \sin p k .
\end{aligned}
$$

Since $\Delta_{\ell}$ is linear, i.e., $c \Delta_{\ell} u(k)=\Delta_{\ell} c u(k)$ and $(\cos p \ell-1)$ and $\sin p \ell$ are constants, multiplying (13) by $(\cos p \ell-1),(14)$ by $\sin p \ell$ and then subtracting the second from the first one, we obtain

$$
\Delta_{\ell}[(\cos p \ell-1) \sin p k-\sin p \ell \cos p k]=(2-2 \cos p \ell) \sin p k .
$$

Now (11) follows from (8) and dividing (15) by $(2-2 \cos p \ell)$.

Similarly, multiplying (13) by $\sin p \ell,(14)$ by $(\cos p \ell-1)$ and then adding them, we arrive

$$
\Delta_{\ell}[\sin p \ell \sin p k-(\cos p \ell-1) \cos p k]=(2-2 \cos p \ell) \cos p k .
$$

Now, (12) follows from (8) and dividing (16) by $(2-2 \cos p \ell)$.

Definition 2.5. Let $u(k)$ and $v(k)$ be complex valued functions defined on $[a, b]$ and $\ell=\frac{b-a}{N}$. The discrete inner product of $u$ and $v$ with respect to $\ell$ is defined as

$$
(u, v)_{\ell}=\left.\ell \Delta_{\ell}^{-1} u(k) v^{*}(k)\right|_{a} ^{b}=\ell \sum_{r=0}^{N-1} u(a+r \ell) v^{*}(a+r \ell) .
$$

The number $\|u\|_{(\ell)}=(u, u)_{\ell}^{1 / 2}=\left\{\ell \sum_{r=0}^{N-1}|u(a+r \ell)|\right\}^{1 / 2}$ is the $L_{\ell}^{2}-$ norm of $u$. We denote $L_{\ell}^{2}(I)$ as the set of all complex valued functions $u(k)$ which are bounded on $I$ and $\|u\|_{\ell}<\infty$. 


\section{Discrete Orthogonal Systems of Functions}

The function $u(k)=\frac{1}{k_{\ell}^{(2)}}$, where $k_{\ell}^{(2)}=k(k-\ell)$, is not bounded on $[0,2 \ell]$, since $u(\ell)=\frac{1}{\ell_{\ell}^{(2)}}=\frac{1}{\ell(\ell-\ell)}=\infty$. Hence, we consider only bounded functions on $I=[a, b]$.

Definition 3.1. Let $S_{\ell}=\left\{\phi_{0}, \phi_{1}, \phi_{2}, \ldots, \phi_{m}\right\}$ be a collection of bounded complex valued functions defined on $I$. If $\left(\phi_{n}, \phi_{m}\right)_{\ell}=0$ whenever $m \neq n$, the collection $S_{\ell}$ is said to be a discrete orthogonal system on $I$ with respect to $\ell$. If in addition, each $u_{n}$ has norm 1 , then $S_{\ell}$ is said to be an orthonormal system.

Example 3.2. Let $I=[0,2 \pi], \ell=\frac{\pi}{N}$ and $N$ is a positive integer. Consider the system of functions $S_{\ell}=\left\{\phi_{0}, \phi_{1}, \phi_{2}, \ldots, \phi_{N}\right\}$, where

$$
\phi_{0}(k)=\frac{1}{\sqrt{2 \pi}}, \quad \phi_{2 n-1}(k)=\frac{\cos n k}{\sqrt{\pi}}, \quad \phi_{2 n}(k)=\frac{\sin n k}{\sqrt{\pi}}, n=1,2,3, \ldots, N
$$

From (11) and (12) for $n=1,2,3, \ldots, N$, we have

$$
\left.\Delta_{\ell}^{-1} \sin n k\right|_{0} ^{2 \pi}=\left.\frac{\sin n(k-\ell)-\sin n k}{2(1-\cos n \ell)}\right|_{0} ^{2 \pi}=0
$$

and

$$
\left.\Delta_{\ell}^{-1} \cos n k\right|_{0} ^{2 \pi}=\left.\frac{\cos n(k-\ell)-\cos n k}{2(1-\cos n \ell)}\right|_{0} ^{2 \pi}=0 .
$$

When $n$ is multiple of $2 \pi$ we find that,

$\left.\Delta_{\ell}^{-1} \sin n k\right|_{0} ^{2 \pi}=\sin n(2 \pi-\ell)+\sin n(2 \pi-2 \ell)+\cdots+\sin n(2 \pi-2 \pi)=0$.

From (8), we have $\Delta_{\ell}^{-1} 1=\Delta_{\ell}^{-1} k_{\ell}^{(0)}=\frac{k_{\ell}^{(1)}}{\ell}$.

Definition 2.5 yields the following relations:

$$
\left\|\phi_{0}\right\|_{\ell}^{2}=\left(\phi_{0}, \phi_{0}\right)_{\ell}=\left.\ell \Delta_{\ell}^{-1} \frac{1}{\sqrt{2 \pi}} \cdot \frac{1}{\sqrt{2 \pi}}\right|_{0} ^{2 \pi}=\left.\frac{\ell}{2 \pi} \Delta_{\ell}^{-1}(1)\right|_{0} ^{2 \pi}=\left.\frac{\ell}{2 \pi} \frac{k_{\ell}^{(1)}}{\ell}\right|_{0} ^{2 \pi}=1 \text {. }
$$

If $\phi_{n}=\frac{\cos n k}{\sqrt{\pi}}$, then we have 


$$
\begin{aligned}
\left\|\phi_{n}\right\|_{\ell}^{2}=\left(\phi_{n}, \phi_{n}\right)_{\ell}=\ell \Delta_{\ell}^{-1} & \left.\frac{\cos ^{2} n k}{\sqrt{\pi} \sqrt{\pi}}\right|_{0} ^{2 \pi}=\left.\frac{\ell}{\pi} \Delta_{\ell}^{-1}\left(\frac{1+\cos 2 n k}{2}\right)\right|_{0} ^{2 \pi} \\
= & \left.\frac{\ell}{\pi} \Delta_{\ell}^{-1} \frac{1}{2}\right|_{0} ^{2 \pi}+\left.\frac{\ell}{2 \pi} \Delta_{\ell}^{-1} \cos 2 n k\right|_{0} ^{2 \pi}=1+0=1 .
\end{aligned}
$$

Similarly, if $\phi_{n}=\frac{\sin n k}{\sqrt{\pi}}$, then we have

$$
\begin{gathered}
\left\|\phi_{n}\right\|_{\ell}^{2}=\left(\phi_{n}, \phi_{n}\right)_{\ell}=\left.\ell \Delta_{\ell}^{-1} \frac{\sin ^{2} n k}{\sqrt{\pi} \sqrt{\pi}}\right|_{0} ^{2 \pi}=1 . \\
\left(\phi_{n}, \phi_{m}\right)_{\ell}=\left.\ell \Delta_{\ell}^{-1} \frac{\cos n k}{\sqrt{\pi}} \cdot \frac{\cos m k}{\sqrt{\pi}}\right|_{0} ^{2 \pi}=\left.\frac{\ell}{2 \pi} \Delta_{\ell}^{-1}(\cos (m+n) k+\cos (m-n) k)\right|_{0} ^{2 \pi}=0 .
\end{gathered}
$$

Also, we obtain

$$
\left(\phi_{n}, \phi_{m}\right)_{\ell}=\left.\ell \Delta_{\ell}^{-1} \frac{\sin n k}{\sqrt{\pi}} \cdot \frac{\sin m k}{\sqrt{\pi}}\right|_{0} ^{2 \pi}=0
$$

and

$$
\left(\phi_{n}, \phi_{m}\right)_{\ell}=\left.\ell \Delta_{\ell}^{-1} \frac{\sin n k}{\sqrt{\pi}} \cdot \frac{\cos m k}{\sqrt{\pi}}\right|_{0} ^{2 \pi}=0 .
$$

From (19)-(24), the system $S_{\ell}$ is a discrete orthonormal system on $I$.

Note. Since

$$
\left.\Delta_{\ell}^{-1} \cos n k\right|_{0} ^{2 \pi}=\left.\frac{\cos n(k-\ell)-\cos n k}{2(1-\cos n \ell)}\right|_{k=0} ^{2 \pi}=0
$$

if $n \ell \neq m 2 \pi$,

$$
\sin n(k-\ell)-\left.\sin n k\right|_{k=0} ^{2 \pi}=0
$$

and

$$
\cos n(k-\ell)-\left.\cos n k\right|_{k=0} ^{2 \pi}=0
$$

for all $n \ell$, we take $\left.\Delta_{\ell}^{-1} \sin n k\right|_{0} ^{2 \pi}=\left.\Delta_{\ell}^{-1} \cos n k\right|_{0} ^{2 \pi}=0$ for all $n=1,2,3, \ldots, N$, where $\ell=\frac{\ell}{N}$.

Example 3.3. Let $\ell=\frac{\pi}{N}, I=[0,2 \pi]$. Then $S_{\ell}=\left\{u_{n}(k)=\frac{e^{i n k}}{\sqrt{2 \pi}}\right\}$, $n=0,1,2, \ldots, N$, is an orthonormal system of complex valued functions on $I$ of period $2 \pi$. 
Proof. Now, from Definition 2.5, we have

$$
\left\|\phi_{n}\right\|_{\ell}^{2}=\left(\phi_{n}, \phi_{n}\right)_{\ell}=\left.\ell \Delta_{\ell}^{-1} \frac{e^{i n k}}{\sqrt{2 \pi}} \frac{e^{-i n k}}{\sqrt{2 \pi}}\right|_{0} ^{2 \pi}=1
$$

and

$$
\begin{aligned}
\left(\phi_{n}, \phi_{m}\right)_{\ell}=\left.\ell \Delta_{\ell}^{-1} \frac{e^{i n k}}{\sqrt{2 \pi}} \frac{e^{-i m k}}{\sqrt{2 \pi}}\right|_{0} ^{2 \pi}=\left.\frac{\ell}{2 \pi} \frac{e^{i(n-m) k}-1}{e^{i(n-m) \ell}-1}\right|_{0} ^{2 \pi}=0 & \\
\text { textif } m \neq n & \in\{1,2,3, \ldots, N\} .
\end{aligned}
$$

Therefore, orthonormality of $S_{\ell}$ follows from (25) and (26).

Theorem 3.4. Let $\left\{\phi_{0}, \phi_{1}, \phi_{2}, \ldots\right\}$ be an orthonormal system on $I$. Define two sequences of functions $\left\{s_{n}\right\}$ and $\left\{t_{n}\right\}$ on $I$ as follows :

$$
\left\{s_{n}(k)\right\}=\left\{\sum_{q=0}^{n} c_{q} \phi_{q}(k)\right\}, \quad\left\{t_{n}(k)\right\}=\left\{\sum_{q=0}^{n} b_{q} \phi_{q}(k)\right\},
$$

where $c_{q}=\left(u, \phi_{q}\right)_{\ell}$ for $q=0,1,2, \ldots$ and $b_{0}, b_{1}, b_{2}, \ldots$ are arbitrary complex numbers. Then for each $n$, we have

$$
\left\|u-s_{n}\right\|_{(\ell)} \leq\left\|u-t_{n}\right\|_{(\ell)} .
$$

Moreover, the equality holds in (28) if and only if $b_{q}=c_{q}$ for $q=0,1,2, \ldots, n$.

Proof. First, we shall prove that

$$
\left\|u-t_{n}\right\|_{(\ell)}^{2}=\|u\|_{(\ell)}^{2}-\sum_{q=0}^{n}\left|c_{q}\right|^{2}+\sum_{q=0}^{n}\left|b_{q}-c_{q}\right|^{2} .
$$

From the linearity of $\Delta_{\ell}^{-1}$ and (17), we have

$$
\left\|u-t_{n}\right\|_{(\ell)}^{2}=\left(u-t_{n}, u-t_{n}\right)_{\ell}=(u, u)_{\ell}-\left(u, t_{n}\right)_{\ell}-\left(t_{n}, u\right)_{\ell}+\left(t_{n}, t_{n}\right)_{\ell} .
$$

Using the linearity of $\Delta_{\ell}^{-1}$, the orthonormality of $\phi_{n}$ and (17), we obtain

$$
\left(t_{n}, t_{n}\right)_{\ell}=\sum_{q=0}^{n}\left|b_{q}\right|^{2}, \quad\left(u, t_{n}\right)_{\ell}=\sum_{q=0}^{n} b_{q} \hat{c}_{q}, \quad\left(t_{n}, u\right)_{\ell}=\sum_{q=0}^{n} \hat{b}_{q} c_{q}
$$

where $\hat{c}_{q}$ and $\hat{b}_{q}$ are complex conjugates of $c_{q}$ and $b_{q}$ respectively. 
Now (29) is derived from (30) and the following relation $\sum_{q=0}^{n}\left|b_{q}-c_{q}\right|^{2}=\sum_{q=0}^{n}\left(b_{q}-c_{q}\right)\left(\hat{b}_{q}-\hat{c}_{q}\right)=\sum_{q=0}^{n}\left|b_{q}\right|^{2}-\sum_{q=0}^{n} b_{q} \hat{c}_{q}-\sum_{q=0}^{n} \hat{b}_{q} c_{q}+\sum_{q=0}^{n}\left|c_{q}\right|^{2}$

Taking $b_{q}=c_{q}$ in (29), we get

$$
\left\|u-s_{n}\right\|_{(\ell)}^{2}=\|u\|_{(\ell)}^{2}-\sum_{q=0}^{n}\left|c_{q}\right|^{2}
$$

Now, (28) follows from (29), (31) and $\sum_{q=0}^{n}\left|b_{k}-c_{k}\right|^{2} \geq 0$.

\section{Discrete Fourier Series of a Function Relative to an Orthonormal System}

Definition 4.1. Let $S_{\ell}=\left\{\phi_{0}, \phi_{1}, \phi_{2}, \ldots, \phi_{M}\right\}$ be an orthonormal system on $I, \ell=\frac{b-a}{2 N}$ and assume that $u$ is a complex valued bounded function on $I$. The notation

$$
u(k)_{\ell} \approx \sum_{n=0}^{M} c_{n} \phi_{n}(k)
$$

will mean that the numbers $c_{0}, c_{1}, c_{2}, \ldots$ are given by the formula

$$
c_{n}=\left(u, \phi_{n}\right)_{\ell}=\left.\ell \Delta_{\ell}^{-1}\left(u(k) \phi_{n}^{*}(k)\right)\right|_{a} ^{b}, \quad n=0,1,2, \ldots
$$

The series in (32) is called the Discrete Fourier Series of $u$ relative to $S_{\ell}$ and the numbers $c_{0}, c_{1}, c_{2}, \ldots$ are called the Discrete Fourier Coefficients of $u$ relative to $S_{\ell}$

Example 4.2. If $I=[0,2 \pi], \ell=\frac{\pi}{N}$ and $S_{\ell}$ is the orthonormal system of trigonometric functions described in (18), then the series obtained by (32) is called discrete Fourier series generated by $u$. In this case, we can write (32) in the form

$$
u(k)_{\ell} \approx \frac{a_{0}}{2}+\sum_{n=1}^{N}\left(a_{n} \cos n k+b_{n} \sin n k\right)
$$


the coefficients being given below.

$$
\begin{gathered}
a_{0}=\frac{\ell}{\pi} \Delta_{\ell}^{-1}\left(\left.u(k)\right|_{0} ^{2 \pi}=\frac{\ell}{\pi} \sum_{r=1}^{\left[\frac{2 \pi}{\ell}\right]} u(2 \pi-r \ell),\right. \\
a_{n}=\left.\frac{\ell}{\pi} \Delta_{\ell}^{-1}(u(k) \cos n k)\right|_{0} ^{2 \pi}=\frac{\ell}{\pi} \sum_{r=1}^{\left[\frac{2 \pi}{\ell}\right]} u(2 \pi-r \ell) \cos (2 \pi-r \ell),
\end{gathered}
$$

and

$$
b_{n}=\left.\frac{\ell}{\pi} \Delta_{\ell}^{-1} u(k) \sin n k\right|_{0} ^{2 \pi}=\frac{\ell}{\pi} \sum_{r=1}^{\left[\frac{2 \pi}{\ell}\right]} u(2 \pi-r \ell) \sin (2 \pi-r \ell) .
$$

The coefficients described in (35)-(37) can be obtained either by closed form or summation form of $\Delta_{\ell}^{-1} u(k)$ depending on $u(k)$ and when $N \rightarrow \infty$ the Discrete Fourier Series converges to Fourier Series.

To obtain orthonormal system and Discrete Fourier Series we develop certain results of $\Delta_{\ell}^{-1}$ on trigonometric functions with $u(k)$.

Lemma 4.3. Let $\ell \neq 0, k>0$ and $p \ell \neq m 2 \pi$. Then we have

$$
\Delta_{\ell}^{-1} \sin (p k+\beta)=\frac{\sin (p(k-\ell)+\beta)-\sin (p k+\beta)}{2(1-\cos p \ell)}
$$

and

$$
\Delta_{\ell}^{-1} \cos (p k+\beta)=\frac{\cos (p(k-\ell)+\beta)-\cos (p k+\beta)}{2(1-\cos p \ell)} .
$$

Proof. Replacing $p k$ by $p k+\beta$ in (2.4) and (12 completes the proof of the lemma.

Definition 4.4. If $\sum_{r \in Z}|u(r \ell)|^{2} \mid<\infty$, where $\mathbb{Z}=\{0, \pm 1, \pm 2, \ldots$,$\} , then$ we say that $f \in L_{\ell}(-\infty, \infty)$ and we denote $\sum_{r \in \mathbb{Z}}|u(r \ell)|^{2}=\left.\Delta_{\ell}^{-1} u(k)\right|_{-\infty} ^{\infty}$.

Remark 4.5. Here, we take $\left.\Delta_{\ell}^{-1} \sin n k\right|_{0} ^{2 \pi}=\left.\Delta_{\ell}^{-1} \cos n k\right|_{0} ^{2 \pi}=0$, when $n \ell=2 m \pi$. 
Theorem 4.6. If $u(k) \in L(I)$ and $\ell=\frac{b-a}{2 N}$, then we have

$$
\left.\lim _{p \rightarrow \infty} \ell \Delta_{\ell}^{-1} u(k) \cos p k\right|_{a} ^{b}=0
$$

Proof. $u(k) \in L(I)$ implies $|u(k)| \leq M$ for all $k \in I$.

Since, $\Delta_{\ell}^{-1} \cos p k=\frac{\cos p(k-\ell)-\cos p k}{2(1-\cos p \ell)}$,

$$
\begin{aligned}
\left|\lim _{p \rightarrow \infty} \ell \Delta_{\ell}^{-1} u(k) \cos p k\right|_{a}^{b} \mid & \leq \ell M\left|\lim _{p \rightarrow \infty} \Delta_{\ell}^{-1} \cos p k\right|_{a}^{b} \mid \\
& \leq \ell M \lim _{p \rightarrow \infty}\left|\frac{4}{2(1-\cos p \ell)}\right| \\
& \leq \ell M \frac{2}{\frac{p^{2} \ell^{2}}{2 !}+\left(\frac{p^{6} \ell^{6}}{6 !}-\frac{p^{4} \ell^{4}}{4 !}\right)+\left(\frac{p^{10} \ell^{10}}{10 !}-\frac{p^{8} \ell^{8}}{8 !}\right) \ldots} .
\end{aligned}
$$

Now (40) follows by taking $p \rightarrow \infty$.

Theorem 4.7. If $u(k) \in L_{\ell}(-\infty, \infty)$, then we have

$$
\left.\ell \Delta_{\ell}^{-1} u(k)\left(\frac{1-\cos p k}{k}\right)\right|_{-\infty} ^{\infty}=\left.\ell \Delta_{\ell}^{-1}\left(\frac{u(k)-u(-k)}{k}\right)\right|_{0} ^{\infty} .
$$

Proof. Since $\frac{1-\cos p k}{k}=0$ at $k=0$ and it is bounded for all $k$,

$$
\left.\ell \Delta_{\ell}^{-1} u(k)\left(\frac{1-\cos p k}{k}\right)\right|_{-\infty} ^{\infty}
$$

exists as $u \in L_{\ell}(-\infty, \infty)$.

By Theorem (4.6), as $u$ is bounded, we have

$$
\left.\ell \Delta_{\ell}^{-1} u(k) \cos p k\right|_{0} ^{\infty}=0
$$

Now, we have

$$
\begin{aligned}
\left.\ell \Delta_{\ell}^{-1} u(k)\left(\frac{1-\cos p k}{k}\right)\right|_{-\infty} ^{\infty}= & \left.\ell \Delta_{\ell}^{-1} u(k)\left(\frac{1-\cos p k}{k}\right)\right|_{0} ^{\infty} \\
& +\left.\ell \Delta_{\ell}^{-1} u(k)\left(\frac{1-\cos p k}{k}\right)\right|_{-\infty} ^{0}
\end{aligned}
$$




$$
\begin{aligned}
= & \left.\ell \Delta_{\ell}^{-1} u(k)\left(\frac{1-\cos p k}{k}\right)\right|_{0} ^{\infty} \\
& +\left.\ell \Delta_{\ell}^{-1} u(-k)\left(\frac{1-\cos p k}{-k}\right)\right|_{0} ^{\infty} \\
= & \left.\ell \Delta_{\ell}^{-1}[u(k)-u(-k)]\left(\frac{1-\cos p k}{k}\right)\right|_{0} ^{\infty} .
\end{aligned}
$$

From (43) we get the required result.

Theorem 4.8. Let $s_{\ell}=\left\{\phi_{0}, \phi_{1}, \phi_{2}, \ldots, \phi_{M}\right\}$ be a system of discrete orthonormal functions defined on $I$, assume that, $f$ is bounded complex-valued function defined on $I$ and suppose that $u(k)_{\ell} \approx \sum_{n=0}^{M} c_{n} \phi_{n}(k)$. Then for $n \leq M$,

(a) The series $\sum_{n=0}^{M}\left|c_{n}\right|^{2}$ converges and satisfies the inequality

$$
\sum_{n=0}^{M}\left|c_{n}\right|^{2} \leq\|u\|_{(\ell)}^{2} \quad \text { (Discrete Bessel's inequality) }
$$

(b) The equation

$$
\sum_{n=0}^{M}\left|c_{n}\right|^{2}=\|u\|_{(\ell)}^{2} \quad \text { (Discrete Parseval's formula) }
$$

holds if and only if $\left\|u-s_{M}\right\|_{(\ell)}=0$, where $\left\{s_{n}\right\}$ is the sequence of partial sums defined by $s_{n}(k)=\sum_{q=0}^{M} c_{q} \phi_{q}(k)$.

Proof. We take $b_{q}=c_{q}$ in (29) and observe that the left member is nonnegative. Therefore $\sum_{q=0}^{n}\left|c_{q}\right|^{2} \leq\|u\|_{(\ell)}^{2}$. This establishes $(a)$.

To prove $(b)$, we again put $b_{k}=c_{k}$ in (29) to obtain

$$
\left\|u-s_{n}\right\|_{(\ell)}^{2}=\|u\|_{\ell}^{2}-\sum_{q=0}^{n}\left|c_{q}\right| .
$$

Part (b) follows at once from this equation. 
Theorem 4.9. Assume that $\left\{\phi_{0}, \phi_{1}, \ldots, \phi_{n}, \ldots, \phi_{M}\right\}$ is a system of discrete orthonormal functions on $I, N$ is very large and $\ell=\frac{b-a}{2 N}$ is very small. Let $\left\{c_{n}\right\}$ be any sequence of complex numbers such that $\sum\left|c_{q}\right|^{2}$ converges. Then there is a function $u$ bounded on $I$ such that:

$(a)\left(u, \phi_{q}\right)_{(\ell)}=c_{q}$ for each $q \geq 0$, and

(b) $\|u\|_{\ell}^{2}=\sum_{q=0}^{M}\left|c_{q}\right|^{2}$.

Proof. Since $\left\{\phi_{q}\right\}$ is discrete orthonormal, we have

$$
\left\|c_{q} \phi_{q}\right\|_{(\ell)}^{2} \leq\left|c_{q}\right|^{2}\left\|\phi_{q}\right\|_{(\ell)}^{2} \leq\left|c_{q}\right|^{2}
$$

Take $u(k)=\sum_{q=0}^{M} c_{q} \phi_{q}(k), k \in I$.

Now

$$
\left(u, \phi_{q}\right)_{\ell}=\left(c_{q} \phi_{q}, \phi_{q}\right)_{(\ell)}=c_{q}\left(\phi_{q}, \phi_{q}\right)_{(\ell)}=c_{q}\left\|\phi_{q}\right\|_{(\ell)}=c_{q} .
$$

Proof of Part (b) follows from (45)

\section{Conclusion}

When $\ell \rightarrow 0$, the Discrete Fourier Series and Discrete Fourier Transforms become usual Fourier Series and the Fourier Transforms. If $\int(\cdot) d x$ is not exist, then we can replace $\int(\cdot)$ by $\ell \Delta_{\ell}^{-1}(\cdot)$ and we can get several applications using discrete Fourier transform and its series using summation form of $\Delta_{\ell}^{-1}$.

\section{Acknowledgments}

Research Supported by National Board for Higher Mathematics, Department of Atomic Energy, Government of India, Mumbai.

\section{References}

[1] H. S. Carslaw , Introduction to the Theory of Fourier's Series and Integrals, 3rd ed. Macmillian, London,1930. 
[2] R. F. Edwards, Fourier Series, A Modern Introduction, Vol. 1. Holt, Rinehart and Winston, New York, 1967.

[3] D. Jackson, Fourier Series and Orthogonal Polynomials. Carus Monograph No. 6, Open Court, New York, 1941.

[4] W. W. Rogosinski, Fourier Series. H. Cohn and F. Steinhardt, translators. Chelsea, New York, 1950.

[5] A. Zygmund, Trigonometrical Series, 2nd ed. Cambridge University Press, 1968.

[6] A. N. Akansu and Poluri, "Walsh-like Nonlinear Phase Orthogonal Codes for Direct Sequences CDMA Communications", IEEE Trans. on Signal Processing, (2007), 38003806.

[7] V. Britanak and K. R. Rao, " The Fast Generalized Discrete Fourier Transforms: A unified Approach to the Discrete Sinusoidal Transforms Computation," Siganal Processing, 79 (1999), 135-150.

[8] M. Golay, " The Merit Factor of Long Low Autocorrelation Binary Sequences," IEEE Trans. on Information Theory, 28 (1982), 543-549.

[9] S. A. Martucci , "Symmetric Convolution and the Discrete Sine and Cosine Transforms," IEEE Trans. on Signal Processing, 42 (1994), 1038-1051.

[10] K. S. Miller and B. Ross, Fractional Difference Calculus in Univalent Functions, Fractional Calculus and Their Applications(Koriyama, 1988), Horwood, Chichester, UK, (1989), 139-152.

[11] N. R. O. Bastos, R. A. C. Ferreira and D. F. M. Torres, Discrete-Time Fractional Variational Problems, Signal Processing, 91(3) (2011), 513-524.

[12] R. A. C. Ferreira and D. F. M. Torres, Fractional h-difference equations arising from the calculus of variations, Applicable Analysis and Discrete Mathematics, 5(1) (2011), 110-121.

[13] M. Maria Susai Manuel, G. B. A Xavier, V. Chandrasekar and R. Pugalarasu, Theory and application of the Generalized Difference Operator of the $\mathrm{n}^{\text {th }}$ kind(Part I), Demonstratio Mathematica,45(1) (2012), 95-106.

[14] M. Maria Susai Manuel , G. Britto Antony Xavier and E. Thandapani , Theory of Generalized Difference Operator and Its Applications, Far East Journal of Mathematical Sciences, 20(2) (2006), 163-171. 
